\section{Sensibilidad no celíaca al gluten. Una patología más que responde al gluten}

\author{
ELIZABETH NAVARRO $^{1, \mathrm{a}}$, MAGDALENA ARAYA ${ }^{2}$
}

\section{Non-celiac gluten sensitivity. Another condition that responds to gluten}

Remission of gastrointestinal and general symptoms after gluten withdrawal has been described in some non-celiac individuals for nearly 30 years. Only recently, efforts have been made to define this entity, now referred to as "non-celiac gluten sensitivity". It includes patients that clinically respond to gluten free diet without exhibiting allergic or autoimmune features to explain such response. Wheat allergy, celiac disease, irritable bowel syndrome and symptoms induced by high FODMAPs (Fermentable, Oligo-, Di-, Mono-saccharides And Polyols) consumption are the main differential diagnoses. The relationship with neuropsychiatric disorders such as schizophrenia and autism has not been demonstrated, but currently it gives ground to great hope in families with affected children. Epidemiology of non-celiac gluten sensitivity is not clear. It is described as more common among women and less common in children. Genetic and immune factors, changes in intestinal microbiota and non-gluten components present in wheat grains are main factors postulated in the pathogenesis of this condition. To date, there are no specific biomarkers for non-celiac gluten sensitivity and diagnosis is reached by excluding other causes of disease. A trial with gluten-free diet and subsequent gluten challenge is the methodology most frequently used to confirm diagnosis.

(Rev Med Chile 2015; 143: 619-626)

Key words: Celiac disease; FODMAPs; Irritable bowel syndrome; Non-celiac gluten sensitivity.

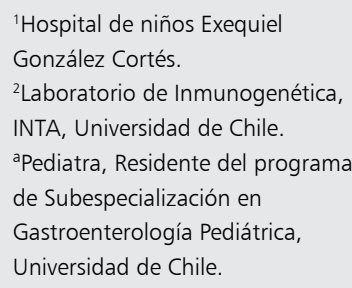

Financiamiento: Este estudio fue financiado por la beca de especialización de la Dra. E. Navarro y el Instituto de Nutrición y Tecnología de los Alimentos (INTA), Universidad de Chile.

Recibido el 16 de diciembre de 2014, aceptado el 10 de abril de 2015.

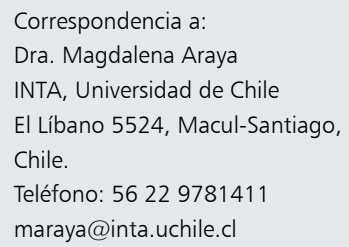

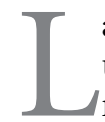

a sensibilidad no celíaca al gluten (SNCG) es una entidad descrita dentro de los desórde$\triangle$ nes asociados al gluten ${ }^{1}$, que está recibiendo progresivamente más atención por parte tanto de profesionales como de la población en general. Se describe que sus síntomas son gatillados por la ingesta de gluten, en ausencia de los anticuerpos y aplanamiento vellositario del intestino delgado típicos de la Enfermedad Celíaca (EC) ${ }^{1}$. En 1978 se describió en Lancet una paciente con diarrea y dolor abdominal que, sin presentar alteraciones en la biopsia duodenal, mejoraba sus síntomas al seguir una dieta libre de gluten (DLG), y éstos reaparecían al reintroducirlo a la $\operatorname{dieta}^{2}$. En la década de los 80 se describió un grupo de mujeres adultas, sin enfermedad celíaca ni alergia al trigo, cuyos síntomas desaparecían con la DLG y reaparecían ante el desafío con gluten ${ }^{3}$. Recientemente, en 2010 Sapone y cols., describieron las características clínicas y diagnósticas de este cuadro, diferenciándolo de la EC por la ausencia de un mecanismo autoinmune involucrado en su patogenia ${ }^{4}$. En 2011, se realizó la primera reunión de expertos en Londres, donde se elaboró un consenso para definir y clasificar las patologías asociadas al gluten ${ }^{1}$. Desde entonces ha aparecido 
un número creciente de publicaciones en el tema que, sin embargo, son insuficientes para entender su epidemiología, fisiopatología, presentación clínica y tratamiento. Las principales limitantes están dadas por la falta de marcadores específicos para hacer el diagnóstico, y la presencia de manifestaciones clínicas que coinciden con las descritas en otras enfermedades, como el síndrome de intestino irritable (SII), enfermedad celíaca (EC) e incluso algunos desórdenes neuro-psiquiátricos como esquizofrenia y autismo. En 2012, se realizó la segunda reunión de expertos, en la que se analizaron los avances en SNCG, ya ampliamente reconocida ${ }^{5}$, a pesar que algunos autores todavía cuestionan si es el gluten u otras proteínas o componentes del grano del trigo los responsables de la sintomatología observada ${ }^{5,6}$.

La evidencia disponible es esencialmente en adultos. Se han publicado dos series de pacientes pediátricos, la de Mastrototaro y cols., en $2012^{7}$ y más recientemente otra por Francavilla y cols. ${ }^{8}$, que describen 12 y 15 casos respectivamente; son estudios prospectivos, que confirmarían que esta condición también existe en la edad pediátrica, con características clínicas similares a las reportadas en adultos. Un estudio en Nueva Zelandia encontró que 5\% de los niños decía evitar los alimentos con gluten para evitar ciertas conductas y/o síntomas gastrointestinales; lamentablemente, los autores no mencionan si había niños celíacos en la muestra9.

Hasta ahora el principal esfuerzo ha sido para separar la SNCG de la EC y la alergia al trigo. La EC es una enfermedad crónica que se manifiesta como un desorden sistémico mediado por fenómenos inmunes y autoinmunes, gatillado por el gluten y prolaminas relacionadas en individuos genéticamente susceptibles. Su inicio es generalmente gradual, existiendo un período de latencia variable de meses a años entre el inicio de la ingesta de gluten y la aparición de la enfermedad. La alergia al trigo es una reacción adversa inmune que puede manifestarse con manifestaciones en la piel, aparato digestivo o respiratorio, en la que los anticuerpos IgE juegan un rol central en la patogénesis; en ella no se describen fenómenos de autoinmunidad. La propuesta de Sapone y cols. ${ }^{1}$ define la SNCG como una reacción adversa al gluten en la que se ha descartado los mecanismos alérgicos (principalmente IgE) y autoinmunes (principalmente tTG2 y EMA).

\section{Epidemiología}

La epidemiología del cuadro aun no es clara. Varios autores ponen énfasis en que la mayoría de las estimaciones de frecuencia se basan en pacientes que se auto diagnostican e inician una dieta libre de gluten sin consulta médica previa, por lo que pueden incluir casos no diagnosticados de EC y de alergia al trigo. No se han identificado factores de riesgo, pero parece ser más común en mujeres adultas jóvenes, con una frecuencia máxima en la cuarta década de la vida, con una relación de 5,4 mujeres: 1 hombre ${ }^{10}$, y con una estimación de prevalencia de 0,63 a $6 \%$ de la población ${ }^{5,11}$.

Hoy se reconoce que la frecuencia de la EC ha aumentado consistentemente en el mundo a lo largo de los últimos 10-20 años, fenómeno que también se observa en nuestro país. La segunda Encuesta Nacional de Salud chilena mostró que $0,75 \%$ de la población es positiva cuando se les mide anticuerpos anti transglutaminasa (tTG2) y la prevalencia de la EC confirmada por biopsia intestinal se estima en $0,6 \%{ }^{16}$. Al igual que en otros países, en nuestro medio el número de pacientes que se autodefinen alérgicos o sensibles al trigo o que hacen dieta sin gluten buscando bajar de peso ha aumentado considerablemente. Esto se ha traducido en que el mercado de los productos libres de gluten y sus consumidores ha aumentado la última década, favoreciendo la confusión y mal manejo de estos cuadros por parte de la población. De allí que consideramos necesario hacer esta revisión, que actualiza y da a conocer el estado del arte de la SNCG.

\section{Factores etiológicos y fisiopatología}

Factores genéticos, la microbiota y la dieta son los factores más frecuentemente invocados para explicarla, de manera parecida a lo que sucede con varias otras enfermedades intestinales que se les reconoce multifactoriales, pero aún no es posible aclarar el peso de cada factor y no se ha logrado diferenciar estas condiciones (Figura 1). Estas patologías incluyen la EC, el SII, la alergia al trigo y la enfermedad inflamatoria intestinal; todas ellas comparten el haber aumentado su frecuencia en el último siglo. Se han postulado tres vías mediante las cuales se puede gatillar la aparición de síntomas: la activación inmune, la acción directa 


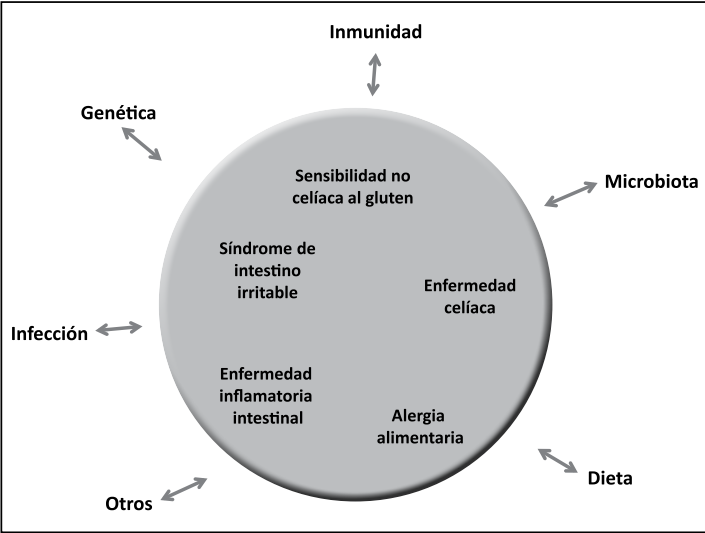

Figura 1. Cuadros clínicos que pueden compartir sintomatología (digestiva y extradigestiva) y potenciales factores patogénicos.

de ciertas moléculas bioactivas contenidas en los alimentos y la distensión del lumen intestinal ${ }^{17}$.

\section{Genética}

En contraste con la EC, en la que un muy alto porcentaje de pacientes son portadores de los haplotipos HLA-DQ2/DQ8 del complejo mayor de histocompatibilidad tipo II, en la SNCG se ha reportado que estos genes están presentes en sólo alrededor de $50 \%$ de los pacientes, cifra mayor que el 30-40\% reportado en población general, pero menor que la descrita entre celíacos ${ }^{1,18}$. En pacientes con SII con predominio de diarrea, se ha descrito una asociación con HLA-DQ2, en ausencia de anticuerpos específicos de $\mathrm{EC}^{19}$.

\section{Microbiota}

En la EC se ha demostrado que existen diferencias en la microbiota de pacientes tratados y no tratados, así como entre tratados y controles sanos. Estas variaciones se basan principalmente en la menor diversidad y distinta composición de la flora bacteriana de pacientes celíacos, en los que la proporción de Bacteroides y Escherichia coli estarían aumentados en los casos no tratados mientras que las especies de Bifidobacterium se encontrarían en menor cantidad, todo ello en relación a lo descrito en controles ${ }^{20}$. Estos datos sugieren que la composición y diversidad de la microbiota jugarían un rol en la enteropatía dependiente de gluten, sin embargo, no hay estudios en pacientes con SNCG propiamente tal. El patrón de infecciones tempranas, que se ha postulado que podría tener un rol en EC, permanece sin datos en la SNCG.

\section{Inmunidad}

Al igual que en otras patologías crónicas digestivas, en la SNCG no se ha logrado establecer los mecanismos inmunológicos que operan en ella. En condiciones fisiológicas, las células dendríticas de la lámina propria son las que promueven tolerancia ante los antígenos que censan en el lumen $^{1}$. Para que el fenómeno de tolerancia ocurra y se mantenga la condición de no-enfermedad, se requiere que los factores inmunes epiteliales y celulares estén maduros y diferenciados. Los péptidos de gliadina pobremente digeridos pueden alterar variadas funciones, tanto en la mucosa intestinal como a nivel sistémico, favoreciendo la autoinmunidad ${ }^{21}$ y la diabetes en $\operatorname{ratas}^{22}$. Por otra parte, las proteínas zonulina y la familia de las claudinas participan en la mantención de la barrera paracelular. Se ha demostrado que la zonulina participa en la patogenia de $\mathrm{EC}^{1}$. En pacientes con SNCG se ha descrito que, en comparación a pacientes celíacos, la expresión de zonulina- 1 y de claudina-1 no serían distintas, pero la expresión de claudina-4 es significativamente mayor, así como la expresión de los receptores toll-like 2 (TLR2) ${ }^{1,6}$. Estos resultados, más otros que evalúan linfocitos intraepiteliales y la presencia de señalizaciones inmunológicas ${ }^{23}$ sugieren que sería la inmunidad innata la que estaría comprometida en la SNCG.

\section{Componentes no gluten de la dieta}

Existe una interesante discusión acerca de si es el gluten $u$ otro de sus componentes los responsables de los síntomas. El trigo fue introducido a la dieta del humano hace unos 10.000 años atrás, lo que desde el punto de vista de la evolución es un tiempo corto para adaptarse a sus componentes; por otro lado, el trigo ha sufrido modificaciones genéticas que han aumentado su contenido de hidratos de carbono, pero también su inmunogenicidad $^{6}$. En la SNCG, el o los gatillantes de 
los eventos fisiopatológicos no necesariamente deben ser los mismos péptidos de gluten que actúan en EC. Estudios in vitro sugieren que inhibidores de la alfa-amilasa/tripsina del trigo (familia de proteínas de bajo peso molecular, altamente resistentes a la proteólisis) podrían tener un importante rol en gatillar la respuesta inmune innata, promoviendo la liberación de citoquinas por parte de monocitos, macrófagos y células dendríticas ${ }^{5,6}$. Desde otro punto de vista, al eliminar el gluten de la dieta también disminuye la ingesta de otros componentes dietarios, que pueden participar en la aparición de sintomatología. Uno de ellos son los FODMAPs ${ }^{24}$, hidratos de carbono de cadena corta (oligosacáridos), di- y monosacáridos y alcoholes relacionados que son pobremente absorbidos en el intestino delgado y que, al pasar al colon son metabolizados por la microbiota intestinal. Dada su alta osmolaridad aumentan el contenido de agua en el lumen, lo que junto a la producción de gas que resulta de la fermentación bacteriana, explica la sintomatología de distensión, dolor abdominal y producción de gases característicos del cuadro que provocan ${ }^{15}$. Hoy día no está claro hasta qué punto, al menos en algunos casos, la sintomatología descrita en la SNCG puede estar confundida con la asociada al alto consumo de FODMAPs, propia de la dieta occidental actual.

\section{Espectro clínico y diagnóstico}

Los síntomas característicos generalmente aparecen horas o pocos días tras la ingesta de gluten, desaparecen con su suspensión y reaparecen al ingerirlos nuevamente ${ }^{5}$. Los síntomas clásicos son similares al SII, como dolor abdominal, náuseas, distensión abdominal, diarrea o constipación; también pueden estar asociados a manifestaciones sistémicas como cefalea, dolor muscular y articular, adormecimiento de brazos y piernas, fatiga crónica, problemas de atención, depresión, baja de peso y anemia (Tabla 1). En niños, las manifestaciones extra intestinales parecen ser menos frecuentes, presentándose en mayor proporción con dolor abdominal y diarrea crónica ${ }^{5,11}$. En comparación con la EC, los pacientes con SNCG presentan compromiso del peso y deficiencias nutricionales con menor frecuencia y severidad, especialmente de vitamina D y hierro ${ }^{25}$.

Hasta ahora, el diagnóstico es de exclusión (Figura 2). No se ha identificado un marcador que identifique la enfermedad y facilite su diagnóstico. Se ha descrito un aumento de los títulos de anticuerpos antigliadina (IgG AGA) en 56\% de los pacientes, tanto adultos como niños; la prevalencia de estos anticuerpos se observa hasta en $81 \%$ de los pacientes celíacos y en 2 a $8 \%$ de los donantes de sangre sanos de la población general ${ }^{5}$. No obstante, los marcadores serológicos de EC, anticuerpos para péptidos deamidados de gliadina (IgG DGP), anticuerpos antitransglutaminasa (IgA TTG) y antiendomisio (IgA EMA) son siempre negativos ${ }^{5}$. El tracto gastrointestinal y su permeabilidad no presentan alteraciones, y los hallazgos histológicos en la mucosa del intestino delgado son menores, con escaso aumento de linfocitos intraepiteliales e infiltración linfocítica de la mucosa, por lo que la clasificación histológica de las mucosas habitualmente es Marsh 0 o 1, a diferencia de la EC en que la lesión de la mucosa

Tabla 1. Síntomas asociados a la sensibilidad no celíaca al gluten

\begin{tabular}{|ll|}
\hline Gastrointestinales & Otras manifestaciones \\
\hline Dolor abdominal & Cefalea \\
\hline Diarrea & Dolor muscular y articular \\
\hline Constipación & Cansancio crónico \\
\hline Distensión abdominal & Adormecimiento de extremidades \\
\hline Flatulencia & Calambres \\
\hline Náuseas-vómitos & Anemia \\
\hline Baja de peso & Trastornos de atención-hiperactividad \\
\hline Estomatitis ulcerativa & Depresión \\
\hline
\end{tabular}




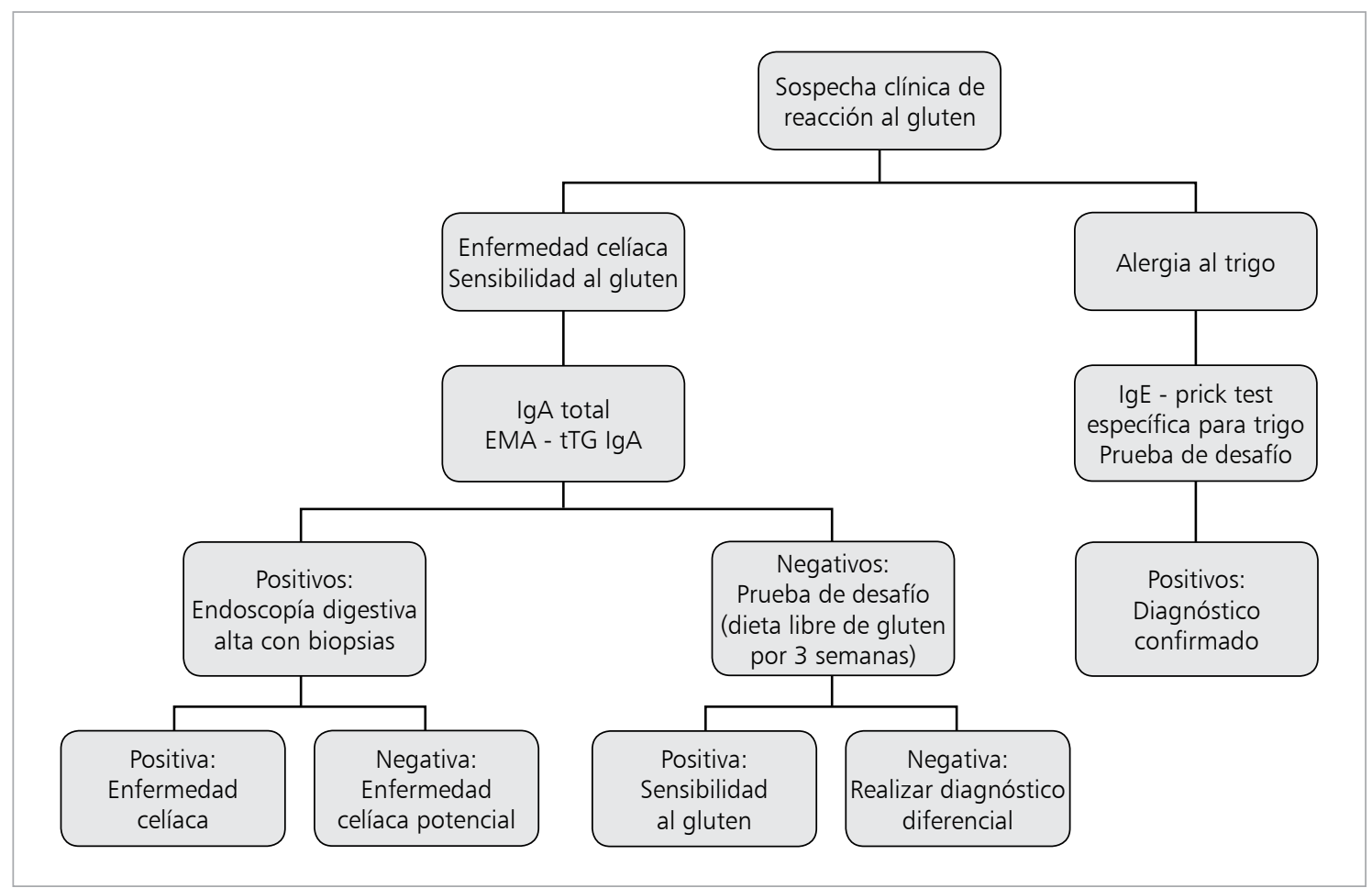

Figura 2. Diagnóstico diferencial de la sensibilidad no celíaca al gluten, enfermedad celíaca y alergia al trigo (adaptado de Sapone et $\left.\mathrm{al}^{4}\right)$.

es Marsh 2 o más. Así, claramente los marcadores usados actualmente en clínica no son útiles como marcadores de $\mathrm{SNCG}^{5,11}$.

Frecuentemente el diagnóstico es sospechado por el paciente. El médico debe basarse en la historia alimentaria, siendo de gran importancia el descartar otros desórdenes relacionados al gluten, como EC y alergia al trigo ${ }^{1}$. La mejoría de los síntomas al eliminar el gluten de la dieta permite plantear el diagnóstico, el cual se confirma con una prueba de desafío con gluten ${ }^{5}$. Se recomienda que esta sea abierta si los síntomas son objetivos como vómitos o diarrea, o en doble ciego si las manifestaciones son subjetivas, como cansancio, cefalea, dolor abdominal. Esta prueba de desafío debe realizarse al menos 3 semanas después de estar en dieta libre de gluten. Sin embargo, existe consenso en que no hay una prueba de desafío estándar y específica para esta condición, y para su diagnóstico más bien se usan adaptaciones de las pruebas que se realizan para las reacciones adversas a alimentos ${ }^{11}$.

\section{SNCG y Síndrome de intestino irritable (SII)}

La relación entre las dos entidades es compleja y merece un párrafo aparte. Ya en 1993 se describió que una proporción de los pacientes con SII alivian sus síntomas al seguir una dieta libre de gluten, sin que presenten la enteropatía típica de la $\mathrm{EC}^{12}$. Vázquez-Roque y cols., han demostrado que pacientes con SII en los que predomina la diarrea, los síntomas mejoran al evitar el gluten. Se ha postulado que este resultado estaría relacionado con la capacidad del gluten de alterar las funciones de barrera en el intestino delgado, principalmente en los pacientes HLA DQ2/DQ8 positivos ${ }^{13}$. En un estudio poblacional realizado en países del norte de Europa, se encontró SII en 16-25\% de los sujetos ${ }^{5,12,13}$; en una sub-muestra de ellos se realizó un desafío con gluten, placebo-control, en doble ciego, y en $28 \%$ se confirmó SNCG. En otro estudio, 276 de 920 individuos (30\%) que sufrían SII según los criterios de Roma III ${ }^{14}$, presentaban además hipersensibilidad a múltiples alimentos, 
incluyendo el trigo ${ }^{5,15}$. Si se confirmara que una alta proporción de los pacientes con SII sufren SNCG, la incidencia de la condición podría ser bastante más alta que la que se piensa hoy.

En pacientes no celíacos, con SII según criterios de Roma III, un estudio reciente randomizado, placebo-control y en doble ciego evaluó si variaban su sintomatología al ingerir gluten. Los pacientes con SII presentaron más dolor, cambios en las heces y sensación de cansancio, sin evidenciar mayor inflamación intestinal o elementos sugerentes de daño o de EC latente ${ }^{26}$. En otro ensayo clínico en doble ciego realizado en 37 pacientes con SNCG y SII (según criterios de Roma III), se evaluó el efecto de una dieta pobre en FODMAPs seguida por un período de dieta libre de gluten; luego se dividieron en 3 tipos de tratamientos (dieta alta en gluten, baja en gluten o placebo) por una semana. En todos los participantes los síntomas disminuyeron con la dieta pobre en FODMAPs. Efectos específicos del gluten se observó en sólo $8 \%$ de los casos y en dos casos no hubo respuesta alguna a la variación del contenido de gluten en la dieta. Los autores concluyeron que el gluten no tuvo efectos específicos ni dosis-dependiente ${ }^{15}$.

\section{SNCG y Desórdenes neuro-psiquiátricos}

En las últimas dos décadas se ha relacionado la SNCG con cuadros como la esquizofrenia y trastornos del espectro autista (TEA), creando confusión y a veces altas expectativas en las familias afectadas. La hipótesis propone que algunos de los síntomas en los TEA estarían causados por péptidos opioides derivados de la incompleta digestión del gluten y caseína de la dieta, los que cruzarían la barrera intestinal (por una permeabilidad intestinal aumentada), pasarían al torrente sanguíneo y de allí pasarían la barrera hemato encefálica, afectando el sistema de opioides endógeno y de neurotransmisión. El exceso de opioides sería el responsable de las alteraciones del comportamiento del paciente. Estudios en niños con TEA han mostrado que tienen niveles de anticuerpos IgG-AGA significativamente mayores que los controles, particularmente aquellos pacientes que además presentan síntomas gastrointestinales ${ }^{5,11}$. Desgraciadamente, la evidencia en el tema es pobre y la eficacia de una dieta libre de gluten (DLG) y caseína no se ha demostrado. Hoy se piensa que sólo un pequeño grupo de estos niños podría beneficiarse con la dieta, pero aun así, el tema es controversial ya que las características clínicas de los pacientes que responden tampoco es clara ${ }^{27}$.

También se ha relacionado la EC y esquizofrenia; la mayoría de los trabajos no demuestran una frecuencia mayor de EC en pacientes esquizofrénicos en comparación a la población general $^{28-30}$. Recientemente, se encontró que 5,5\% de estos pacientes mostraba niveles elevados de anticuerpos IgG-antitransglutaminasa y $23 \%$ de ellos anticuerpos IgG-antigliadina, pero la EC se confirmó en sólo $2 \%$ de ellos ${ }^{31}$. Sin embargo, un estudio en Inglaterra encontró una frecuencia 3 veces mayor que la esperada ${ }^{32}$ y hay casos en que los síntomas de esquizofrenia mejoraron con la dieta sin gluten ${ }^{33}$. Es interesante notar que en los pacientes neuropsiquiátricos la mayor parte de los anticuerpos antitransglutaminasa son tTG-6, indicadores de neuroinflamación, más que de un trastorno asociado al gluten, en que se eleva la tTG-2 $2^{5,11}$.

\section{Manejo}

El único tratamiento disponible es la DLG, sin embargo, no se han descrito complicaciones en pacientes con SNCG no tratados, especialmente en relación a patologías autoinmunes o al desarrollo de linfoma intestinal, como se observa en la EC. Tampoco se han demostrado beneficios ni efectos negativos en la salud en pacientes según sigan o no una dieta libre de gluten ${ }^{34}$. El impacto de la sintomatología tiene consecuencias variables en los pacientes; en algunos la calidad de vida se ve muy afectada por la sintomatología, aumentando la carga de enfermedad a los servicios de salud, de manera similar a lo que ocurre en el SII. Es evidente la necesidad de entender mejor esta entidad y desarrollar guías que permitan identificar y diagnosticar eficientemente a los individuos con SNCG, que se beneficiarán de una dieta de restricción.

\section{Referencias}

1. Sapone A, Bai J, Ciacci C, Dolinsek J, Green P, Hadjivassiliou, et al. Spectrum of gluten-related disorders: consensos on new nomenclature and classification. BMC Medicine: 2012; 10: 13. 
2. Ellis A, Linaker B. Non-celiac gluten sensitivity? Lancet: 1978; 1: 1358-9.

3. Cooper B, Holmes K, Thompson F, Allan N, Cooke W. Gluten-sensitive diarrhea without evidence of celiac disease. Gastroenterology: 1980; 79: 801-6.

4. Sapone A, Lammers K, Mazzarella G, Mikhailenko I, Carteni M, Casolaro V, et al. Differential mucosal IL-17 expression in two gliadin-induced disorders: gluten sensitivity and the autoinmune enteropathy celiac disease. Int Arch Allergy Inmunol 2010; 152: 75-80.

5. Guandalini S1, Polanco I. Nonceliac gluten sensitivity or wheat intolerance syndrome? J Pediatr 2015; 166 (4): 805-11.

6. Nijeboer P, Bontkes H, Mulder C, Bouma G. Non-celiac gluten sensitivity. Is it in the gluten or the grain? J Gastrointestin Liver Dis 2013; 22 (4): 435-40.

7. Mastrototaro L, Castellaneta S, Gentile A, Fontana C, Tandoi E, Dellate S, et al. Gluten sensitivity in children: clinical, serological, genetic and histological description of the first paediatric series. Dig Liver Dis 2012; Suppl 4 (44): S241- S57.

8. Francavilla R, Cristofori F, Castellaneta S, Polloni C, Albano V, Dellatte S, et al. Clinical, serologic and histologic features of gluten sensitivity in children. J Pediatr 2014; 164 (3): 463-7.

9. Tanpowpong P, Ingham T, Lampshire P, Kirchberg F, Epton M, Crane J, et al. Coeliac disease andgluten avoidance in New Zealand children. Arch Dis Child 2012; 97: 12-6.

10. Volta U, Bardella M, Calabrò A, Troncone R, Corazza G. An italian prospective multicenter Surrey on patients suspected of having non-celiac gluten sensitivity. BMC Medicine 2014; 12: 85.

11. Czaja-Bulsa G. Non coeliac gluten sensitivity-a new disease with gluten intolerance. Clinical Nutrition: 2014 (article in press).

12. Arranz E, Ferguson A. Intestinal antibody pattern of celiac disease: occurrence in patients with normal jejunal biopsy histology. Gastroenterology 1993; 104: 1263-72.

13. Vázquez-Roque $M$, Camilleri $M$, Smyrk T, Murray J, Marietta E, ONeill J, et al. A controlled trial of gluten-free diet in patients with irritable bowel syndrome-diarrhea: effects on bowel frequency and intestinal function. Gastroenterology 2013; 144: 903-11.

14. Carroccio A, Mansueto P, Iacono G, Soresi M, D’Alcamo A, Cavataio F, et al. Non-celiac wheat sensitivity diagnosed by double-blind placebo-controlled Challenger: exploring a new clinical entity. Am J Gastroenterol 2012; 107: 1898-906.

15. Biesiekierski J, Peters S, Newnham E, Rosella O, Muir J, Gibson P. No effects of gluten in patients with self-reported non-celiac gluten sensitivity alter dietary reduction of fermentable, poorly absorbed, short-chain carbohydrates. Gastroenterology 2013; 145: 320-8.

16. MINSAL. Encuesta Nacional de Salud ENS Chile: 20092010.

17. Gibson P. Food intolerance in functional bowel disorders. J Gastroenterol Hepatol 2011; 26 (suppl 3): 128-31.

18. Monsuur A, Wijmenga C. Understanding the molecular basis of celiac disease: what genetic studies reveal. Ann Med 2006; 38: 578-91.

19. Sapone A, Lammers K, Casolaro V, Cammarota M, Giuliano M, De Rosa M, et al. Divergence of gut permeability and mucosal inmune gene expresión in two gluten-associated conditions: celiac disease and gluten sensitivity. BMC Med 2011; 9: 23.

20. Marietta E, Murray J. Animal models to study gluten sensitivity. Semin Inmunophatol 2012; 34 (4): 497511.

21. Ziegler A, Schmid S, Huber D, Hummel M, Bonifacio E. Early infant feeding and risk of developing type 1 diabetes-associated autoantibodies. JAMA 2003; 290 (13): 1721-8.

22. Visser J, Lammers K, Hoogendijk A, Boer M, Brugman $S$, Beijer-Liefers $S$, et al. Restoration of impaired intestinal barrier function by the hydrolysed casein diet contribuyes to the prevention of type 1 diabetes in the diabetes-prone Bio Breeding rat. Diabetologia 2010; 53 (12): 2621-8.

23. Whanschaffe U, Ullrich R, Riecken E, Schulzke J. Celiac disease-like abnormalities in a subgroup of patients with irritable bowel syndrome. Gastroenterology 2001; 121: 1329-38.

24. Cuomo R, Andreozzi P, Zito F, Passananti V, De Carlo G, Sarnelli G. Irritable bowel syndrome and food interaction. World J Gastroenterol 2014; 20 (27): 8837-45.

25. Kabbani T, Vanga R, Leffler D, Villafuerte-Galvez J, Pallav K, Hansen J, et al. Celiac disease or non-celiac gluten sensitivity? An aprroach to clinical differential diagnosis. Am J Gastroenterol 2014; 109: 741-6.

26. Biesiekierski J, Sci B, Newhnham E, Irving P, Barrett J, Haines $M$, et al. Gluten causes gastrointestinal symptoms in subjects without celiac disease: a double-blind randomized placebo-controlled trial. Am J Gastroenterol 2011; 106: 508-14.

27. Buie T. The relationship of autism and gluten. Clin Ther 2013; 35: 578-83.

28. Khoshbaten M, Rostami Nejad M, Sharifi N, Fakhari A, Golamnejad M, Hashemi SH, et al. Celiac disease in patients with chronic psychiatric disorders. Gastroenterol Hepatol Bed Bench 2012 Spring; 5 (2): 90-3.

29. Eaton WW, Byrne M, Ewald H, Mors O, Chen CY, 
Agerbo E, et al. Association of schizophrenia and autoimmune diseases: linkage of Danish National Registers. Am J Psychiatry 2006; 163: 521-8.

30. West J, Logan RF, Hubbard RB, Card TR. Risk of schizophrenia in people with coeliac disease, ulcerative colitis and Crohn's disease: a general population-based study. Aliment Pharmacol Ther 2006; 23: 71-4. Prevalence of celiac disease and gluten sensitivity in the United States clinical antipsychotic trials of intervention effectiveness study population.
31. Cascella NG, Kryszak D, Bhatti B, Gregory P, Kelly DL, Mc Evoy JP, et al. Schizophr Bull 2011; 37 (1): 94-100.

32. John M. Celiac disease: Is it a cause of schizophrenia? BMJ 2004; 328: 438-9.

33. De Santis A, Addolorato G, Romito A, Caputo S, Giordano A, Gambassi G, et al. Schizophrenic symptoms and SPECT abnormalities in a coeliac patient: regression after a gluten-free diet. J Intern Med 1997; 242: 421-3.

34. Lundin K. Non-celiac gluten sensitivity-why to worry? BMC Medicine 2014; 12: 86. 